\title{
Sensors for waveform control in a Lorentz force based actuator system for turbulence research
}

\author{
F.Seidler ${ }^{1}$, M. Schiek ${ }^{1}$, W. Silex ${ }^{1}$, R. Heil ${ }^{1}$, S. van Waasen ${ }^{1,2}$, D. Abel $^{3}$ \\ 1. Central Institute of Engineering, \\ Electronics and Analytics, Electronic \\ Systems (ZEA-2), \\ Forschungszentrum Jülich GmbH, \\ Jülich, Germany \\ 2. Faculty of Engineering, \\ Communication \\ Systems Department, \\ University of Duisburg-Essen, \\ Duisburg, Germany \\ 3. Institute of Automatic Control, \\ Department of Mechanical \\ Engineering, \\ RWTH Aachen University, \\ Aachen, Germany
}

f.seidler@fz-juelich.de

\begin{abstract}
This work discusses the use of analog light barrier and Hall sensors for waveform control in an actuator system for turbulence research. The system shall produce up to $80 \mathrm{~Hz}, 80 \mathrm{~mm}$ wavelength running surface wave with amplitudes from 50 to 1000 $\mu \mathrm{m}$. To ensure both, waveform and amplitude, the control algorithm requires real time amplitude measurements of all 20 actuators. Techniques are described to extend the limited measurement range of analog light barriers and to reduce impact of mechanical disturbances on the measurement process by placement and compensation. Also a small bar permanent magnet together with Hall sensors to track position are employed and a method for reducing impact of the strong magnetic stray fields affecting Hall sensors in this actuator system is developed.
\end{abstract}

Keywords-Light barrier; Hall sensor; Turbulence control; VHCF experiments; Drag reduction.

\section{INTRODUCTION}

DFG Research group FOR 1779 studies drag reduction in turbulent flow by transversal wavy surface oscillations. Within this group we are developing an improved Lorentz force based actuator system for further experiments in turbulence control exploring beyond previous experimental results [1]. With a very similar approach, also very high cycle fatigue (VHCF) experiments requiring constant monitoring of the actuator movements have been successfully carried out [2]. More recent results include the development of a smooth transition system for sine signals used for this actuator system on the basis of direct digital synthesis (DDS) [7].

Due to strong coupling of the 20 actuators by the aluminum surface, the system has to be controlled to ensure waveform and amplitude of the running wave. To apply recently developed iterative learning control to the system [3], amplitudes of all actuators need to be measured precisely. The wind tunnel

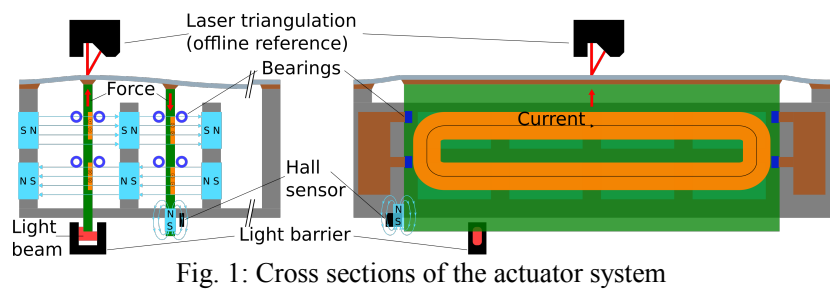

experiments do not allow measurements from above. For the setup commercially available sensors and impact of placement on their performance are characterized. Furthermore techniques to compensate for the most important disturbances for analog light barrier sensors and Hall sensors used in combination with small permanent magnets are shown.

The first section of this article explains operating conditions and working principle of the actuator system as far as this is relevant to the operation of the sensors discussed here. The final section features major conclusions and an outlook on future developments.

\section{SET-UP AND OPERATING CONDITIONS}

The actuator system consists of a frame made largely of $5 \mathrm{~mm}$ aluminum bars with fixed permanent magnets as shown in the cross sections in Fig. 1. Between these magnets PCB bars with embedded 80 winding air core coils are inserted. They are guided by small ball bearings at both ends. The top of both PCB bars and outermost aluminum frame are glued to an aluminum sheet of $0.3 \mathrm{~mm}$ thickness. Applying a current to the coils will result in a force moving the sheet. Although bearings and glue constrain the movement of the individual actuators to almost exclusively the z-direction, due to mechanical imperfections of bearings and actuator bars, i.e. elasticity and slack, actuators tilt and bend in $\mathrm{x}$-direction. While this movement is very limited, the only guidance that prevents tilting along the y-axis is the aluminum sheet. However since the sheet is not perfectly homogeneous and there are limits to manufacturing precision its reset force is not completely symmetrical. The amplitude differences at actuation frequencies of $80 \mathrm{~Hz}$ typically range from $5 \%$ to $20 \%$ of the total amplitude.

This movement is captured through tracking the position of the actuator bars by the sensors discussed below. This information is needed to develop and run a feedback wave control system making a wider wave parameter range available and for comparison with numerical flow simulations. However these sensors are not pre-calibrated. Therefore we need a reference which is provided by a Keyence laser triangulation sensor. It tracks the position of one spot on the surface and can easily be placed over any actuator bar and quickly be removed after calibration and before the start of the experiment. This is 

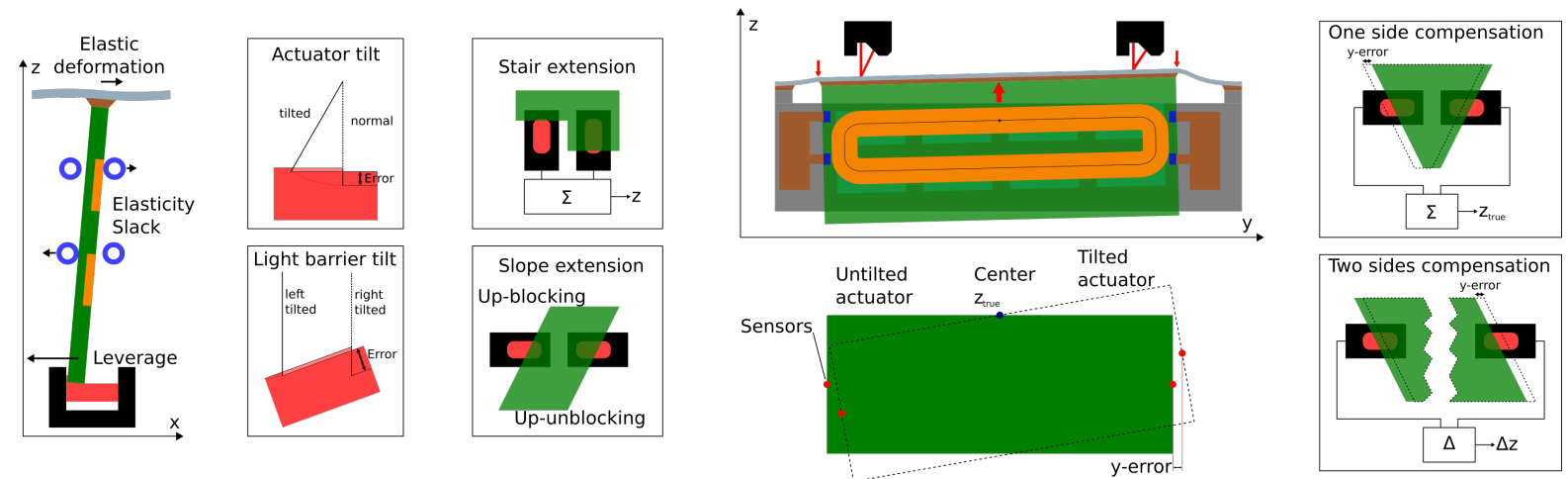

Fig. 2: Range extension, tilting motions and their impact on light barriers. The bottom of the actuator is constrained to move $\Delta x=1 \mathrm{~mm}$ right and left.

With height $h$ of the bar, actuator tilt will account for $\Delta z=h-\sqrt{h^{2}+\Delta x^{2}}=5.5 \mu \mathrm{m}$. Light barrier tilt $\alpha$ of $5^{\circ}$ will result in $\Delta z=2 \Delta x \cdot \tan (\alpha)=175 \mu \mathrm{m}$

important because the system is to be used in wind tunnel experiments. We are strictly required to keep the whole set-up including online sensors well below the level of the aluminum sheet not to disturb the flow above it which is the subject of the experiment. The PCB bars are placed with $10 \mathrm{~mm}$ spacing to accommodate for our goal of reaching a shorter wavelength than with the previous set-up (20 mm spacing). This limits the selection of sensors and is one of the main reasons not to use a larger number of the reference sensors for online tracking.

In addition to these mechanical constraints, as the setup is made for laboratory use only it has no magnetic shielding. This allows for the magnetic field from the drive coils to extend well outside the mechanical dimensions of the set-up. We call this the drive coil stray field. It is important to keep this in mind when considering sensors based on magnetic fields for this set-up.

\section{LIGHT BARRIER SENSOR}

The analog light barrier registers how much of the light beam emitted from one side reaches the other [4]. When inserting a sheet of non-transparent material with a straight edge and clipping the light beam, we can detect the position of the edge of the sheet by measuring the sensor output and linking it to a reference position measurement. The maximum distance the sensor can cover depends on the spatial extent of the beam. Unfortunately the maximum range of $0.6 \mathrm{~mm}$ of a single light barrier [4] used this way is not sufficient for our task. However by using a slope we can extend this distance trading precision for range. For our purposes a 1:4 slope would be sufficient, extending $0.6 \mathrm{~mm}$ of linear range to $2.4 \mathrm{~mm}$. Alternatively multiple light barriers with a stair-like edge, such that as soon as one barrier is completely blocked another one takes over, are used. In practice this is done by summing all analog signals using an operational amplifier such that in the overlap region one light barrier fades out when the other fades in creating an approximately linear behavior.

Geometrical considerations show that movement in $\mathrm{x}$ direction is largest at the bottom of the actuator bars. Deviations caused by this effect are small but can be exacerbated if the light barrier is not mounted perfectly orthogonal to the actuator bar. Placing the light barriers on the side of the setup instead, close to the bearings minimizes the amount of $\mathrm{x}$-direction movement and associated disturbances of the measurement.
Tilting in y direction requires compensation with a second sensor on the same actuator bar for the slope configuration. The second sensor may be placed on the same side on the same kind of slope or it may be placed on the opposite end of the actuator on the opposite kind of slope shown in the schematic Fig. 2. For one sided compensation the error in $z$ due to y-direction movement $\Delta z_{y}$ will result in opposite change of output for both sensors while actual movement $z$ will result in the same change. Assuming linearity this means the output of the sensors $u_{1}$ and $u_{2}$ added will yield:

$$
u_{1}+u_{2}=z+\Delta z_{y}+z-\Delta z_{y}=2 z .
$$

Similarly for two sides with the movements $z_{1}$ and $z_{2}$ we get:

$$
\begin{aligned}
u_{1}+u_{2}= & z_{1}+\Delta z_{y}+\left(-z_{2}\right)-\Delta z_{y} \\
& =z_{1}-z_{2}=\Delta z,
\end{aligned}
$$

the true position difference between the ends, which can be used to find e.g. $z_{1}$.

\section{HALL SENSOR}

For distance measurement a Hall sensor and a short bar magnet are combined [5]. Due to its operation principle the Hall sensor only measures magnetic field in one direction. The sensor is placed as close as possible to the bar magnet for the largest possible signal per movement. Its measurement axis is oriented orthogonal to the axis of the bar magnet shown in the schematic Fig. 3. Since the magnetic field of a bar magnet is parallel to its axis at the equator [6], there the Hall sensor reading is zero. Moving the magnet up or down results in a change of direction and magnitude of the magnetic field at the position of the Hall sensor and corresponding change of the sensor signal. When
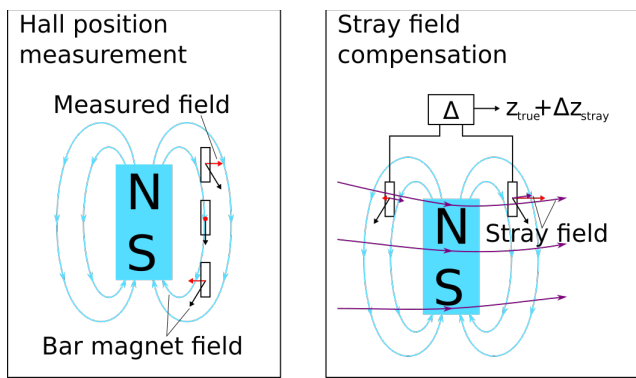

Fig. 3: Hall position measurement and stray field compensation. 


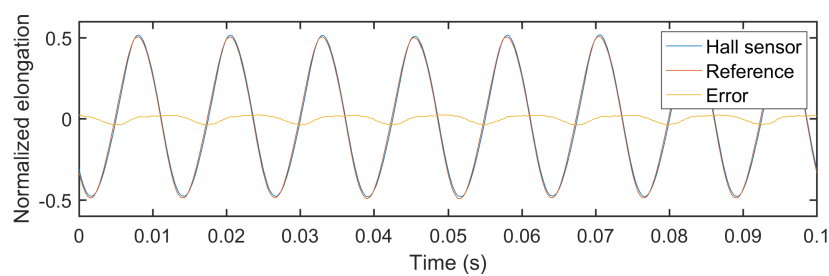

Fig. 4: Light Barrier position tracking using slope at $80 \mathrm{~Hz}$.

moving only between the magnetic poles, this change is monotonic but not linear. It can be calibrated to position values using the reference sensor.

Tilt in $\mathrm{x}$-direction leads to a similar situation as the one of light barriers and can be neglected if the sensor is placed close to the bearings in proper orientation. Tilt in y-direction is even less problematic, since the y-component of the field does not contribute to the measurement. However we still want to measure it. For realization magnets and sensors at both ends of the actuator bar can be placed.

The Hall sensor also picks up the drive coil stray field. To avoid this pick-up they should be placed as far away from the drive coils as possible since it decays cubically [6]. However we are limited by the existing structure of the actuator system and forced to place the sensors within the stray field. It and the actuator movement are same frequency sinusoids under typical operating conditions. Therefore the stray field component cannot be removed by filtering. Nevertheless by placing two Hall sensors on opposite sides of the bar magnet we are able to cancel out the stray field to a certain extent. How well this works depends on how much the stray field component parallel to the measurement axis of the Hall sensors changes from one side of the magnet to the other as shown in the schematic Fig. 3. Assuming symmetrical placement of the sensors and a symmetrical field around the bar magnet, meaning equal magnitude but opposite direction bar magnet field, we get

$$
\begin{gathered}
\left(B_{1, \text { stray }}+B_{1, \text { bar }}\right)-\left(B_{2, \text { stray }}+B_{2, \text { bar }}\right) \\
=\Delta B_{\text {stray }}+2 \cdot B_{\text {bar }},
\end{gathered}
$$

where $B$ are the components of the fields indicated at the indicated positions in measurement direction and $\Delta B_{\text {stray }}$ the change of the stray field from Hall sensor 1 to Hall sensor 2. This technique is useful as long as $2 \cdot \Delta B_{\text {stray }}<$ $\min \left(B_{1, \text { stray }}, B_{2, \text { stray }}\right)$.

Despite these improvements we cannot avoid that the performance of the Hall sensor deteriorates in unfavorable stray field conditions. Unfortunately our goal is just such a scenario: at high phase shift of $\pi / 4$ between neighboring actuators coil currents differ more than in our current tests without phase shift. In addition actuators move less, impeded by their neighbors. Larger $\Delta B_{\text {stray }}$ and reduced amplitudes have to be expected.

\section{EXPERIMENTAL RESULTS}

For testing of placement and compensation schemes the sensors were mounted to a test set-up with only three actuator bars which is easier to modify. As reference the position of two points of the surface over one bar was tracked with reference

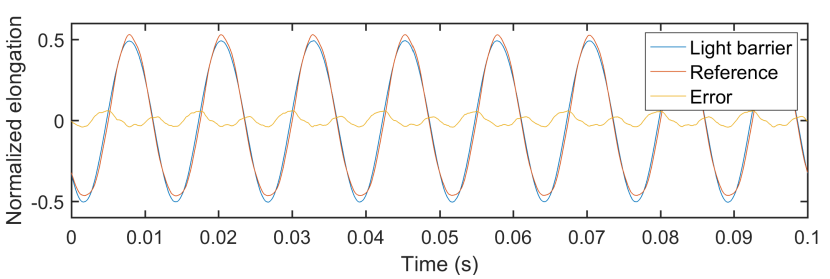

Fig. 5: Hall sensor position tracking at $80 \mathrm{~Hz}$.

sensors and the expected sensor reading was calculated. Graphs in Fig. 4 and 5 compare the results. The error is well below $10 \%$ in both cases.

\section{CONCLUSION}

The sensors and the techniques developed for their application in FOR1779 research group provide fast, precise, and reliable readings to allow control of the amplitude and waveform. Due to operating conditions around the actuator system placement of sensors and use of appropriate compensation techniques is crucial for success.

Future research will focus on including the sensors in advanced feedback control and modeling. For modeling neural networks or other machine learning techniques will be investigated. Further automation of the calibration process will improve usability. In the next development step the amplitude and waveform will be dynamically adapted to changing flow conditions making use of the smooth parameter transition developed by Völkel et al. [7]. The presented techniques can be used in other smart surface applications or in very high cycle fatigue experiments.

\section{ACKNOWLEDGMENT}

The support of this research by the Deutsche Forschungsgemeinschaft DFG within the framework of FOR 1779 is gratefully acknowledged.

\section{REFERENCES}

[1] W. Li, W. Jessen, D. Roggenkamp, M. Klaas, W. Silex, M. Schiek, and W. Schröder, "Turbulent drag reduction by spanwise traveling ribbed surface waves," European Journal of Mechanics-B/Fluids, vol. 53, pp. 101-112, 2015.

[2] S. Stille, M. Schiek, W. Silex, T. Beck, S. van Waasen, and L. Singheiser, "Novel approach for endurance testing of riblet-structured thin sheets under realistic loading conditions in active drag-reduction systems," Journal of Testing and Evaluation, vol. 45(4), 2016.

[3] M. Dueck, D. Abel, S. van Waasen, and M. Schiek, "Transversal surface wave control by gain switching iterative learning improving research on active turbulent flow control," Control Conference (AUCC), 2015 5th Australian, IEEE, pp. 300-305, November 2015.

[4] Vishay Semiconductor GmbH, TCST1103 datasheet, Rev. A5, 1999.

[5] A. Latham, W. Bussing, "Slide-by sensing for long-stroke applications using allegro angle sensors," Allegro MicroSystems LLC, 2016.

[6] D.J. Griffiths, "Introduction to Electrodynamics (3rd ed.)," Pearson Education, pp 243-267, 2007.

[7] S. Völkel, R.Heil, M. Schiek, M. Schlösser, W. Silex, S. van Waasen, "Smooth wavelength transition in a Lorentz force based actuator system for turbulence research", IEEE SENSORS 2017 conference proceedings, in press. 